\title{
SULFs in human neoplasia: implication as progression and prognosis factors
}

\author{
Caroline Bret ${ }^{1,2,3}$, Jérôme Moreaux ${ }^{1}$, Jean-François Schved ${ }^{2,3}$, Dirk Hose ${ }^{4,5}$ and Bernard Klein ${ }^{1,3^{*}}$
}

\begin{abstract}
Background: The sulfation pattern of heparan sulfate chains influences signaling events mediated by heparan sulfate proteoglycans located on cell surface. SULF1 and SULF2 are two endosulfatases able to cleave specific 6-O sulfate groups within the heparan chains. Their action can modulate signaling processes, many of which with key relevance for cancer development and expansion. SULF1 has been associated with tumor suppressor effects in various models of cancer, whereas SULF2 dysregulation was in relation with protumorigenic actions. However, other observations argue for contradictory effects of these sulfatases in cancer, suggesting the complexity of their action in the tumor microenvironment.
\end{abstract}

Methods: We compared the expression of the genes encoding SULF1, SULF2 and heparan sulfate proteoglycans in a large panel of cancer samples to their normal tissue counterparts using publicly available gene expression data, including the data obtained from two cohorts of newly-diagnosed multiple myeloma patients, the Oncomine Cancer Microarray database, the Amazonia data base and the ITTACA database. We also analysed prognosis data in relation with these databases.

Results: We demonstrated that SULF2 expression in primary multiple myeloma cells was associated with a poor prognosis in two independent large cohorts of patients. It remained an independent predictor when considered together with conventional multiple myeloma prognosis factors. Besides, we observed an over-representation of SULF2 gene expression in skin cancer, colorectal carcinoma, testicular teratoma and liver cancer compared to their normal tissue counterpart. We found that SULF2 was significantly over-expressed in high grade uveal melanoma compared to low grade and in patients presenting colorectal carcinoma compared to benign colon adenoma. We observed that, in addition to previous observations, SULF1 gene expression was increased in T prolymphocytic leukemia, acute myeloid leukemia and in renal carcinoma compared to corresponding normal tissues. Furthermore, we found that high SULF1 expression was associated with a poor prognosis in lung adenocarcinoma.

Finally, SULF1 and SULF2 were simultaneously overexpressed in 6 cancer types: brain, breast, head and neck, renal, skin and testicular cancers.

Conclusions: SULF1 and SULF2 are overexpressed in various human cancer types and can be associated to progression and prognosis. Targeting SULF1 and/or SULF2 could be interesting strategies to develop novel cancer therapies.

\section{Background}

Heparan sulfate proteoglycans (HSPGs) are negativelycharged proteins located at a high cell density on various cell types or released into the extracellular matrix. As HSPGs bind a large diversity of molecules: growth factors (GF), cytokines, chemokines, morphogens, matrix ligands and cell surface molecules, they are involved in

\footnotetext{
* Correspondence: bernard.klein@inserm.fr

${ }^{1}$ INSERM U847, Institut de Recherche en Biothérapie, CHRU de Montpellier, France

Full list of author information is available at the end of the article
}

cell signaling as co-receptors [1]. The complexity of the heparan sulfate (HS) chains is based on modifications as epimerisation, de-acetylation and sulfation. These phenomenons strongly influence the ligand binding properties of HSPGs and define the concept of "HS code". The sulfation pattern in glucosamines and uronic acids is dynamically regulated during many cellular processes, generating diversity of the chains and thus diversity of binding. Such mechanisms are regulated by sulfotransferases involved in the biosynthesis of HS. Another class of enzymes is also implicated at the extracellular level:

\section{Biomed Central}


the sulfatases sulfatase 1 (SULF1) and sulfatase 2 (SULF2). Initially cloned in 2002 [2], these secreted enzymes display endoglucosamine 6-sulfatase activity. The expression of the genes encoding these enzymes is developmentally regulated. In murine model, simultaneous disruption of both SULF1 and SULF2 leads to perinatal lethality and developmental defects underlying overlapping and essential roles during development [3]. However, SULF1-deficient mice did not present any abnormal phenotype whereas SULF2-knock-out mice displayed a small but significant reduction in litter size and body weight, and a hydrocephalus at birth resulting in a life span shorter than 2 weeks [4].

Owing to the involvement of HSPGs as coreceptors of cell communication molecules, the role of these HSPG modifying enzymes in human tumorigenesis is actively investigated. Despite similar substrate specificity, SULF1 has mainly tumor suppressor functions whereas SULF2 presents tumor promoting functions. In this article, we focused on recent and challenging data describing the implication of SULF1 and SULF2 in human neoplasia.

\section{Methods}

\section{Databases}

SULF1 and SULF2 gene expression levels in normal or malignant human tissues or cell lines were obtained from the Oncomine Cancer Microarray database (http:// www.oncomine.org) [5], the Amazonia database (http:// amazonia.montp.inserm.fr/) [6] and the ITTACA database (Integrated Tumor Transcriptome Array and Clinical data Analysis) developed by the Institute Curie Bioinformatics group and the Institute Curie, CNRS UMR144 (http://bioinfo-out.curie.fr/ittaca/) [7]. Gene expression data only obtained from a single study using the same methodology were compared. All data were log transformed, median centered per array and the standard deviation was normalized to one per array.

\section{Primary myeloma cells}

Multiple Myeloma cells (MMC) were purified from 206 patients with newly-diagnosed MM after written informed consent was given at the University hospitals of Heidelberg (Germany) or Montpellier (France). The study was approved by the ethics boards of Heidelberg University and Montpellier University. After Ficoll-density gradient centrifugation, plasma cells were purified using anti-CD138 MACS microbeads (Miltenyi Biotech, Bergisch Gladbach, Germany). Microarray experiments were performed in DNA microarray platform of the Institute of Research in Biotherapy at the Montpellier University Hospital (France) http://irb.montp.inserm.fr/ en/index.php? page $=$ Plateau\&IdEquipe $=6$. The. CEL files and MAS5 files have been deposited in the ArrayExpress public database, under accession number E-MTAB-362.
We also used Affymetrix data of a cohort of 345 purified MMC from previously untreated patients from the Arkansas Cancer Research Center (ACRC, Little Rock, AR). These data are publicly available via the online Gene Expression Omnibus (Gene Expression Profile of Multiple Myeloma, accession number GSE2658, http:// www.ncbi.nlm.nih.gov/geo/).

\section{Statistical analysis}

Statistical comparisons were done with Student t-tests. The event free or overall survival of subgroups of patients was compared with the log-rank test and survival curves computed with the Kaplan-Meier method. The prognostic values of parameters were compared with univariate or multivariate Cox analysis. Statistical tests were performed with the software package SPSS 12.0 (SPSS, Chicago, IL).

\section{Results and discussion}

\section{Tumor suppressor functions of SULF1}

Expression of SULF1 mRNA can be detected in several normal human tissues, as observed by MorimotoTomita et al. [2] in a panel of 24 tissue types, the highest levels being found in testes, stomach, skeletal muscle, lung, and kidney. SULF1 down-regulation has been described in human primary tumorous samples and/or cell lines in ovarian cancer [8-10], hepatocellular carcinoma [11], breast cancer [12], gastric cancer [12], kidney cancer [12], prostatic stromal cells from benign prostatic hyperplasia samples [13] and head and neck squamous cell carcinoma (SCCHN) cell lines [14]. This low expression level is mostly explained by epigenetic silencing mediated by hypermethylation of the promoter of the gene encoding SULF1 $[9,12]$.

Considering that HSPG sulfation pattern drives in part cell communication molecule binding [15-17], a loss of SULF1 expression is expected to disrupt the effects of these cell communication molecules during malignancies. It has been observed that this down-regulation results in increased sulfation of HS chains and could produce the stabilization of ternary receptor complexes, leading to an increased in GF signalling, as described for heparin-binding epidermal growth factor-like growth factor (HB-EGF), fibroblast growth factor 2 (FGF2) or amphiregulin in ovarian cancer [8], SCCHN cell lines [14], hepatocellular carcinoma [18] or in breast cancer [19]. This modulation of GF effects can affect major events including proliferation of cancer cells. A forced expression of SULF1 induced growth inhibition of neck squamous cell carcinoma cell lines in vitro[14]. A marked reduction of the growth of myeloma or breast cancer cell lines was observed in severe combined immunodeficient (SCID) mice when injected cell lines were transfected with SULF1 cDNA [20,21]. Forced 
expression of SULF1 also significantly delayed the growth of hepatocellular carcinoma cell lines xenografts in nude mice [22].

These different models also argued the role of SULF1 as an inhibitor of motility, invasion and angiogenesis and as a protein linked to drug-induced apoptosis. Hepatocyte growth factor (HGF)-mediated motility and invasion were attenuated in SCCHN cell lines displaying an overexpression of this sulfatase [14]. Xenografts derived from SULF1-expressing carcinoma cells presented a significantly reduced ability of vascular HS to promote a stable complex between FGF2 and its specific receptor with an inhibition of angiogenesis as a result. The down-regulation of SULF1 in human umbilical vein endothelial cells (HUVECs) could increase vascular endothelial growth factor (VEGF)-induced angiogenic response [21]. In hepatocellular carcinoma (HCC), SULF1 enhanced the induction of apoptosis by the histone deacetylase (HDAC) inhibitors in vitro[22]. The doxorubicin and apicidin-induced apoptosis was significantly increased of in HCC cell lines expressing SULF1. In addition, the anti-tumor effects of these drugs were enhanced in vivo when a xenograft was established from SULF1-expressing HCC [23]. SCCHN-transfected cell lines displayed significant growth inhibition concomitant with an increased sensitivity to staurosporine- and cisplatin-induced apoptosis [14].

Altogether, these data suggest that the widespread SULF1 down-regulation in cancer might be an important contributor to the carcinogenesis process.

\section{SULF2, a protumorigenic endosulfatase}

The implication of SULF2 in cancer was less studied than that of SULF1. However, most of the studies documented a protumorigenic role of SULF2 at the opposite of that of SULF1. Lemjabbar-Alaoui et al. [24] observed an induction of SULF2 expression in human lung adenocarcinoma and squamous cell carcinoma with a mean increase of 3-fold compared to normal lung. They could obtain a loss of the transformed phenotype of lung carcinoma cell lines when silencing SULF2 expression with short-hairpin RNA (sh-RNA). The knock-out of SULF2 in these cell lines also resulted in a decreased tumor formation when grafted to nude mice. Besides, SULF2 was shown to modulate the bioavailability of wingless-type MMTV integration site family (Wnt) ligands, a critical canonical cascade reactivated in several tumors [25]. An up-regulation of SULF2 mRNA was also observed in human or murine breast cancers compared to normal breast tissues [26]. SULF2 was up-regulated in primary HCC samples, as well as in HCC cell lines [11]. It resulted in an activation of mitogen-activated protein kinase (MAPK) and v-akt murine thymoma viral oncogene homolog 1 (Akt) pathways with an increased cell growth in vitro and in vivo. In multiple myeloma (MM), we had previoulsy reported an overexpression of SULF2 gene in primary myeloma cells of newly-diagnosed myeloma compared to normal bone marrow plasma cells [27]. In this study, we demonstrate for the first time that SULF2 expression in primary multiple myeloma cells (MMCs) ("absent" versus "present" Affymetrix call) was associated with a poor prognosis in two independent large cohorts of myeloma patients at diagnosis (206 patients in the cohort of Heidelberg-Montpellier and 250 patients in the cohort of Little-Rock previously described [28], Figure $1 \mathrm{~A}$ and $1 \mathrm{~B}$ ). Patients with SULF2 $2^{\text {absent }}$ MMCs had a significant increased overall survival compared with patients with SULF2 $2^{\text {present }}$ MMCs $(p=0.007$ in the Heidelberg-Montpellier cohort and $p=0.03$ in the Little-Rock cohort), after high-dose therapy and stem cell transplantation. In a Cox proportional hazard model (Table 1), the absence or the presence of SULF2 ( $p=$ 0.007 , hazard ratio $=4.08)$ and ISS stage $(p=0.001$, hazard ratio $=1.73$ ) were independently predictive for overall survival $(p=0.02$ and $p=0.001$, respectively). If SULF2 expression was tested together with classical prognostic factors, i.e., serum albumin and serum beta 2 microglobulin (b2M), SULF2 expression $(p=0.03)$ and b2M $(p=0.0001)$ remained independent prognostic factors. SULF2 expression was an independent prognostic factor of spiked MMSET expression, that is an indicator of $\mathrm{t}(4 ; 14)$ translocation [29] $(p=0.023$ and $p=0.028$ respectively), of the myeloma high risk score (HRS) [30] ( $p=0.01$ and $p=0.002$ respectively), of the growth proliferation index [31] $(p=0.01$ and $p=0.0001$ respectively), of the IFM score [32] ( $p=0.01$ and $p=0.0001$ respectively) and of CD200 expression [33] ( $p=0.02$ and $p=0.05$ respectively). Investigating the SULF2 expression in the 7 groups of the molecular classification [34] of MM, SULF2 was significantly overexpressed in the hyperdiploid group and significantly underexpressed in the groups of patients characterized by Cyclin D1 or MAF translocations (Figure 2). We analyzed the correlation between SULF1 or SULF2 expression and HS proteoglycans expression in our cohort of myeloma patients (syndecan 1-4, glypican 1-6, CD44 isoforms containing the alternatively spliced exon v3, agrin, betaglycan, perlecan, serglycin and testican 1-3)[27]. No significant correlation was found between the expression of the SULFs and of their potential HS proteoglycan targets in MM. When we analyzed the correlation between the expression of the sulfatases and of the genes encoding the transporters and the enzymes involved in HS and chondroïtine sulfate biosynthesis pathway [27], we did not found any correlation for SULF2 but we observed a correlation between SULF1 expression and GALK1 (galactokinase 1) and SLC2A9 (solute carrier family 2, facilitated glucose transporter member 9) expression. 


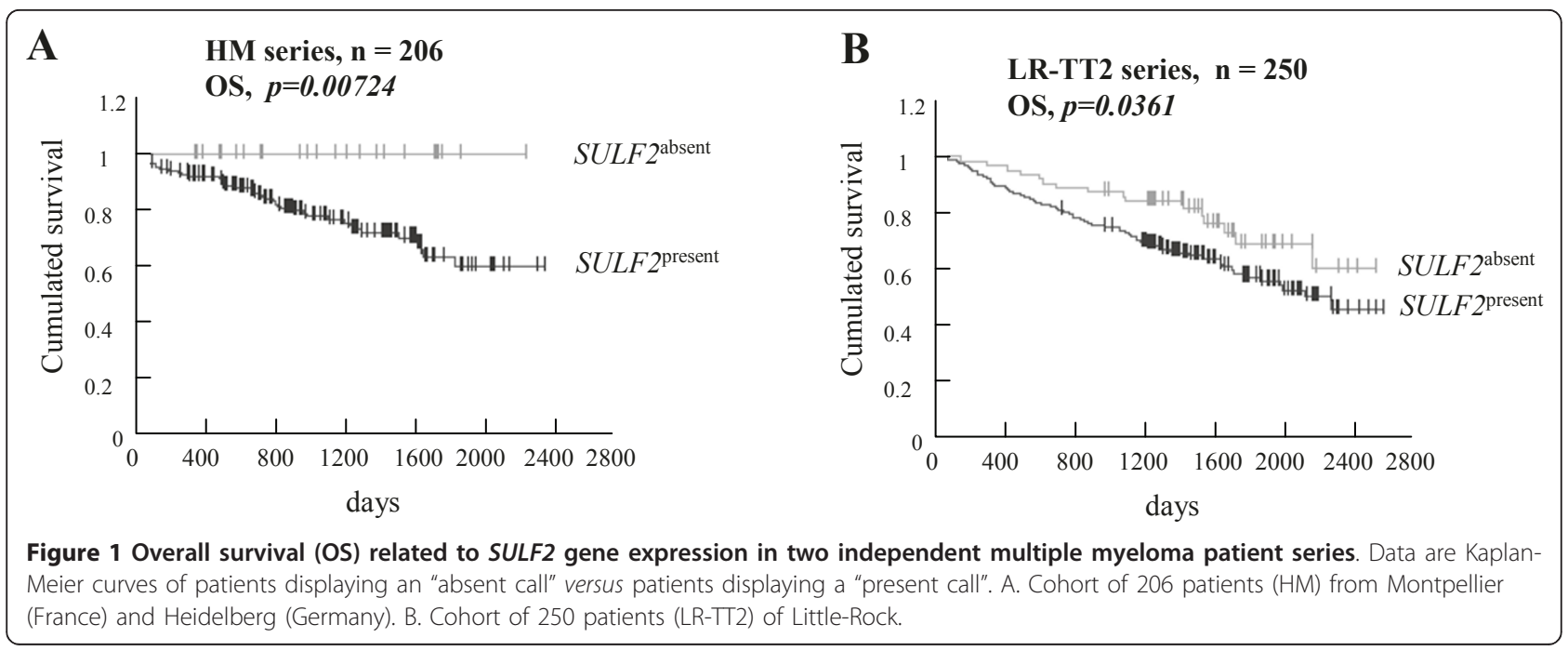

In HCC model, sh-RNA targeting SULF2 induced an inhibition of HCC cell lines proliferation and migration in vitro. In nude mice, SULF2 could significantly promote HCC xenograft growth. Besides, forced expression of this enzyme increased glypican-3 expression level, this membrane-anchored HSPG being involved in Wnt pathway, FGF signaling and cell proliferation [35]. Moreover, in patients with HCC, high levels of SULF2 were associated with a worse prognosis [11]. In human pancreatic carcinoma, the SULFs are up-regulated and it has been observed that the silencing of SULF2 could lead to an inhibition of Wnt signalling and of cell growth [36]. In order to explore the pathogenesis of glioblastoma, Johansson et al. generated a mouse glioma model using a recombinant Moloney murine leukemia virus encoding the platelet-derived growth factor Bchain and intra-cerebrally injected in newborn mice [37]. Using expression profiling, they identified markers of gliomagenesis, SULF2 appearing among the candidate cancer-causing genes.

In addition to its contribution during tumor growth development, SULF2 could be implicated in resistance to cancer treatment, as recently suggested by Moussay et al. [38]. A comparison of gene expression profiles of sensitive and resistant clones of chronic lymphocytic leukemia obtained from patients treated by fludarabine was performed. Together with $v$-myc myelocytomatosis viral oncogene homolog (MYC), SULF2 transcripts were significantly over-represented in cells of patients resistant to fludarabine.

Recently, SULF2 gene expression was investigated in a large panel of cancer samples, using the ONCOMINE microarray database (https://www.oncomine.org 4.3 research edition) [39]. Rosen et al. demonstrated an overexpression of SULF2 in several cancers including brain, breast, tongue and renal carcinomas [39]. In addition to these observations, we found that other cancer types displayed an over-representation of SULF2 gene expression compared to their tissue counterpart: skin $(p=2.26 E-4$ and $p=1 E-3[40])$, colorectal carcinoma $(p=0.02[41])$, testicular teratoma $(p=6 E-3[42])$ and liver cancer $(p=1.9 E-4$ and $p=2 E-3$ [43]). Using the ITTACA database (Integrated Tumor Transcriptome Array and Clinical data Analysis, http://bioinfo-out.curie. fr/ittaca/)[7] and the AMAZONIA database [6], we searched to identify if SULF2 expression could be associated with tumor progression in these cancer types. Interestingly, we found that SULF2 was significantly over-expressed in high grade uveal melanoma compared to low grade $(p=0.03$, Figure 3A). Furthermore, SULF2 was also overexpressed in patients presenting colorectal carcinoma compared to benign colon adenoma ( $p=$ 0.001, Figure 3B).

These different data lend support for a protumorigenic effect of SULF2 overexpressed by many tumor cell types.

\section{Challenging observations concerning SULF1 and SULF2 in cancer}

Using the ONCOMINE microarray database, Rosen et al. shown that, in contrast to the down-regulation of SULF1 reported in various tumor models, SULF1 gene expression was increased in a large range of cancers compared to their corresponding normal tissues [39]. SULF1 was clearly over-expressed in adrenal carcinoma, brain cancer, breast carcinoma, colon adenocarcinoma, skin carcinoma, esophageal and gastric cancers, head and neck cancers, lung cancer, mesothelioma, pancreatic cancer, sarcoma and germ line/testicular cancer [39]. In addition, we found that other cancer types displayed an over-representation of SULF1 gene expression: T prolymphocytic leukemia $(p=0.01$ [44]), acute myeloid leukemia $(p=0.004[45])$ and renal carcinoma $(p<0.001$ 
Table 1 Univariate and multivariate proportional hazards analyses linking SULF2 expression to prognosis in HM cohort

\begin{tabular}{|c|c|c|c|}
\hline \multicolumn{4}{|c|}{ HM cohort (OS) } \\
\hline & Pronostic variable & Proportional hazard ratio & P-value \\
\hline Univariate & SULF2 & 4.08 & 0.007 \\
\hline Cox analysis & ISS & 1.73 & 0.001 \\
\hline Multivariate & SULF2 & 3.65 & 0.028 \\
\hline Cox analysis & ISS & 1.70 & 0.001 \\
\hline Univariate & SULF2 & 4.08 & 0.007 \\
\hline \multirow[t]{2}{*}{ Cox analysis } & b2M & 1.10 & 0.0001 \\
\hline & Alb & 1.60 & 0.04 \\
\hline Multivariate & SULF2 & 3.49 & 0.03 \\
\hline \multirow[t]{2}{*}{ Cox analysis } & b2M & 1.10 & 0.0001 \\
\hline & Alb & 1.35 & 0.24 \\
\hline Univariate & SULF2 & 4.08 & 0.007 \\
\hline Cox analysis & HRS & 2.30 & 0.002 \\
\hline Multivariate & SULF2 & 4.11 & 0.01 \\
\hline Cox analysis & HRS & 2.31 & 0.002 \\
\hline Univariate & SULF2 & 4.08 & 0.007 \\
\hline Cox analysis & MS group & 2.14 & 0.001 \\
\hline Multivariate & SULF2 & 3.84 & 0.023 \\
\hline Cox analysis & MS group & 1.97 & 0.028 \\
\hline Univariate & SULF2 & 4.08 & 0.007 \\
\hline Cox analysis & IFM score & 3.09 & 0.0001 \\
\hline Multivariate & SULF2 & 4.29 & 0.014 \\
\hline Cox analysis & IFM score & 3.22 & 0.0001 \\
\hline Univariate & SULF2 & 4.08 & 0.007 \\
\hline Cox analysis & GPI & 2.21 & 0.0001 \\
\hline Multivariate & SULF2 & 4.47 & 0.011 \\
\hline Cox analysis & GPI & 2.25 & 0.0001 \\
\hline Univariate & SULF2 & 4.08 & 0.007 \\
\hline Cox analysis & MYEOV & 3.16 & 0.05 \\
\hline Multivariate & SULF2 & 3.71 & 0.026 \\
\hline Cox analysis & MYEOV & 2.76 & 0.08 \\
\hline Univariate & SULF2 & 4.08 & 0.007 \\
\hline Cox analysis & CD200 & 2.05 & 0.03 \\
\hline Multivariate & SULF2 & 3.86 & 0.02 \\
\hline Cox analysis & CD200 & 1.03 & 0.05 \\
\hline
\end{tabular}

Univariate analyses were done to screen for prognostic variables linked to SULF2 expression using Cox proportional hazards regression. The Cox model was also used for multivariate analysis to identify the most significant variables related to survival (OS): ISS (international staging system), b2M (beta2 microglobulin), Alb (Albumin), HRS (High Risk Score), MS group (MMSET group), IFM score (IFM score), GPI (Growth Proliferation Index), MYEOV and CD200. P-values are in bold and italic when a significant result was obtained $(p<0.05)$.

[46]). These data challenge the above concept of SULF1 as a tumor suppressor effector. Using the ITTACA database, we aimed to identify if SULF1 expression could be associated with tumor progression or bad prognosis in cancers. Indeed, we found that high SULF1 expression was associated with a poor prognosis in lung adenocarcinoma (Figure 4) [47]. Although SULF1 was overexpressed in breast cancer compared to its normal counterpart $[39,48,49]$, we did not found any significant association between SULF1 expression and survival in

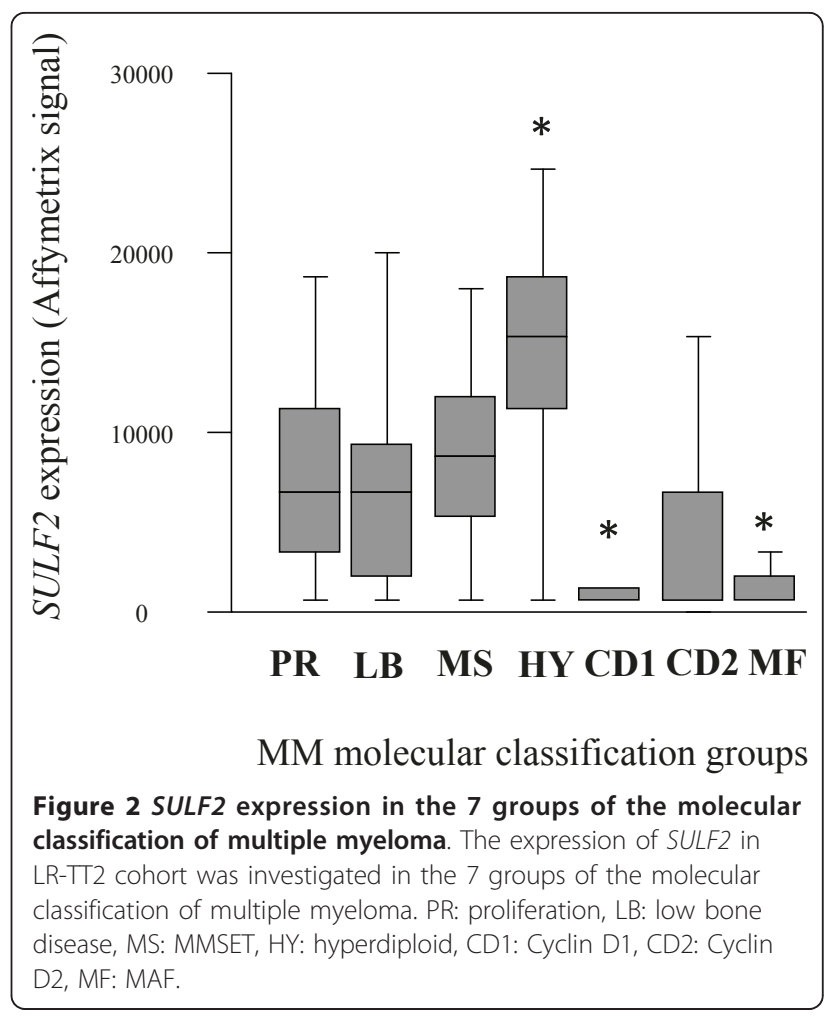

breast cancer using data from two independent studies (data not shown).

Some studies have brought some explanations about this contradictory contribution to carcinogenesis. In pancreatic cancer cells, the expression of SULF1 in xenograft models was associated with a markedly reduced growth potential, but with an increase in the basal invasiveness of these cells [50]. Recently, Sahota and Dhoot [51] demonstrated in quail model the possibility of alternative splicing of SULF1 gene, generating a novel shorter isoform called SULF1B. While the previously described SULF1 (SULF1A) enhanced Wnt signaling, SULF1B inhibited Wnt signaling and promoted angiogenesis. Such splicing has not been yet described in human tissues but could be of interest, in particular in cancer development. In mutiple myeloma, we previously observed an overexpression of SULF1 by bone marrow stromal cells, whereas primary malignant plasma cells did not express the gene encoding for this sulfatase. Besides, SULF1 was expressed by some human myeloma cell lines (HMCLs), emphasizing that these HMCLs can express environment genes, making it possible to escape from environment dependence [27].

Whereas SULF2 is considered as being associated with protumorigenic effects, as reviewed above, a few challenging studies argue for a tumor suppressor effect of this protein. In contrast with our report that SULF2 expression in primary malignant plasma cells is associated 
A

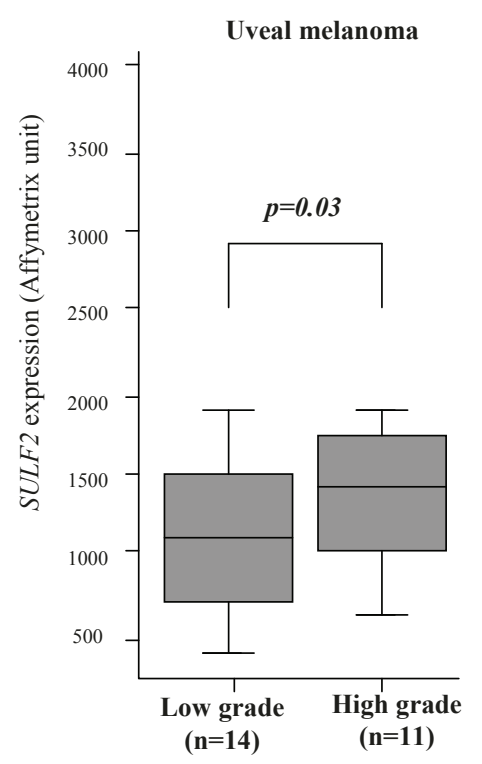

B

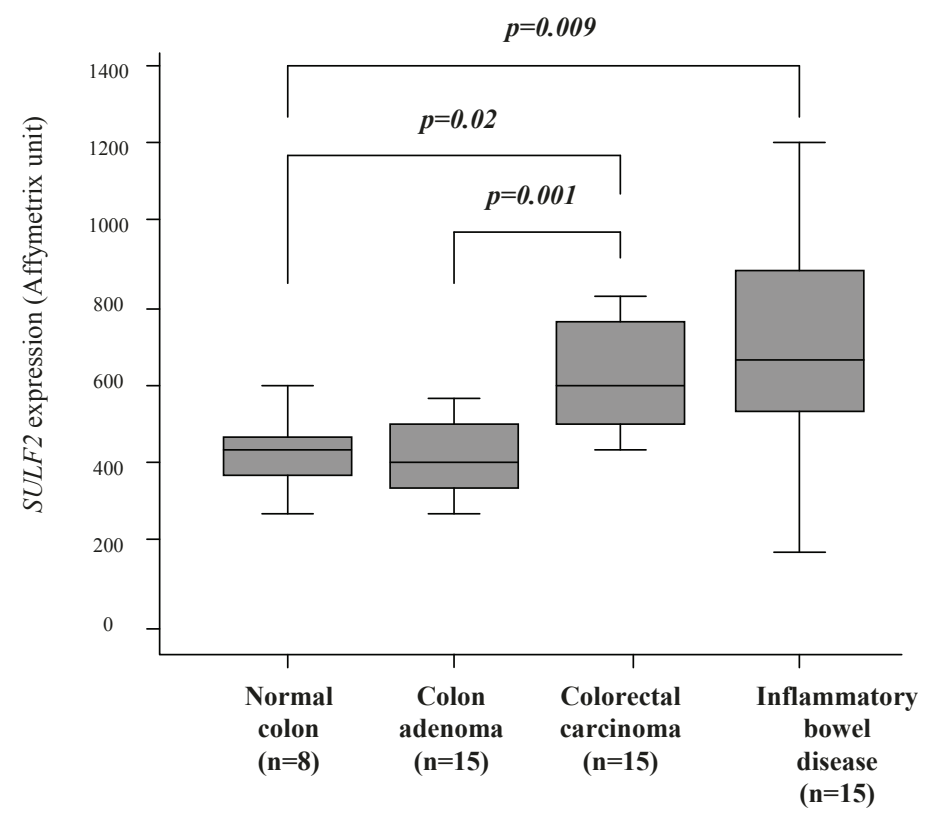

Figure 3 Association between SULF2 expression and progression in various cancers. A. SULF2 gene expression in uveal melanoma [55]. B. SULF2 gene expression in samples of normal colon, adenoma, colorectal carcinoma and inflammatory bowel disease [41]. $P$ values are indicated in each panel.

with poor overall survival [27], Dai et al. [20] observed that a forced expression of SULF2 reduced the growth of myeloma cell lines in SCID mice. Thus, they concluded to a similar action of SULF1 and SULF2 on myeloma cells expansion through the modification of HS

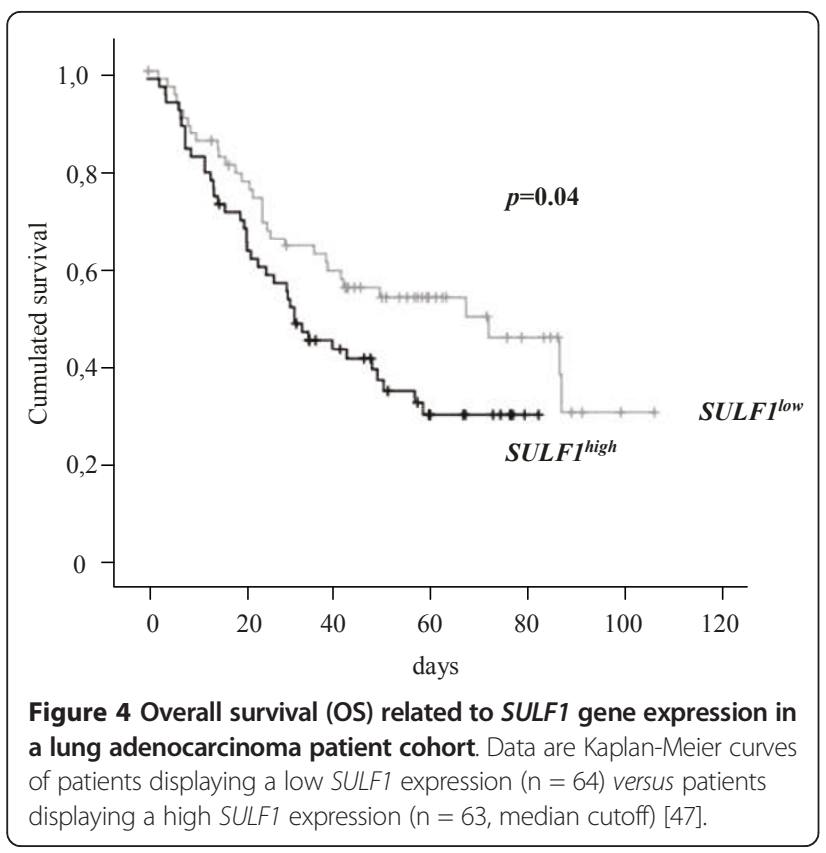

sulfation pattern and its consequence in medullar microenvironment.

In addition to this in vivo observation, two studies demonstrated that SULF2 is induced by p53 tumor suppressor. Adamsen et al. [52] firstly suggested that SULF2 was a putative p53 target gene in colon cancer cells treated by 5 -fluorouracil. Inducible p 53 knockdown cell lines of multiple cancer types were generated by Chau et al. [53] and their gene expression profiles were compared to the initial cell lines. This method led to the identification of downstream targets of p53. SULF2 was found to be a direct transcriptional target of p53 that could bind to the SULF2 promoter, in particular in the context of DNA-damaged-induced senescence, in accordance with the observation of Adamsen.

Interestingly, SULF1 was overexpressed in 6/7 cancer types characterized by SULF2 overexpression compared to normal tissue counterparts (Table 2). Several HS proteoglycans have been identified so far - syndecan 1-4, glypican 1-6, CD44 isoforms containing the alternatively spliced exon v3, agrin, betaglycan, perlecan, serglycin and testican 1-3 - and their gene expression could be evaluated by microarrays [27]. In cancer samples displaying an overexpression of SULF1 and/or SULF2 compared to their normal counterparts, we systematically observed on overexpression of at least one HS proteoglycans (Table 2). The functional consequences of the 
Table 2 Expression of genes encoding SULF1, SULF2 and heparan sulfate proteoglycans in human cancer samples in comparison with their normal counterpart

\begin{tabular}{|c|c|c|c|c|c|c|c|c|c|c|c|}
\hline \multirow[b]{2}{*}{$\begin{array}{c}\text { Cancer } \\
\text { sample type }\end{array}$} & \multirow[b]{2}{*}{ Datasets } & \multicolumn{10}{|c|}{ Gene overexpressed in cancer samples in comparison to their normal tissue counterpart } \\
\hline & & SULF1 & SULF2 & $\begin{array}{c}\text { Syndecan } \\
1-4\end{array}$ & $\begin{array}{c}\text { Glypican } \\
1-6\end{array}$ & $\begin{array}{l}\text { CD44 isoforms } \\
\text { containing the } \\
\text { alternatively spliced } \\
\text { exon v3 }\end{array}$ & Agrin & Betaglycan & Perlecan & Serglycin & $\begin{array}{c}\text { Testican } \\
1-3\end{array}$ \\
\hline Leukemia & 33 & $\underline{\text { Yes }}$ & No & No & No & No & No & No & Yes & Yes & Yes \\
\hline $\begin{array}{l}\text { Adrenal } \\
\text { cancer }\end{array}$ & 2 & Yes & No & No & No & No & No & No & No & No & No \\
\hline Brain cancer & 23 & Yes & Yes & Yes & Yes & Yes & Yes & Yes & Yes & Yes & No \\
\hline Breast cancer & 44 & Yes & Yes & Yes & No & Yes & No & No & No & No & Yes \\
\hline Colon cancer & 12 & Yes & No & No & No & $\underline{\text { Yes }}$ & No & No & No & No & No \\
\hline $\begin{array}{l}\text { Esophageal } \\
\text { cancer }\end{array}$ & 4 & Yes & No & Yes & Yes & Yes & Yes & Yes & Yes & Yes & No \\
\hline Gastric cancer & 5 & Yes & No & No & No & No & No & No & Yes & No & Yes \\
\hline $\begin{array}{l}\text { Head \& Neck } \\
\text { cancer }\end{array}$ & 5 & Yes & Yes & Yes & Yes & Yes & No & No & Yes & Yes & No \\
\hline Liver cancer & 4 & No & Yes & No & No & No & No & No & No & No & No \\
\hline Lung cancer & 16 & Yes & No & No & No & No & Yes & No & No & No & Yes \\
\hline Mesothelioma & 3 & Yes & No & No & No & No & No & No & No & No & No \\
\hline $\begin{array}{c}\text { Pancreatic } \\
\text { cancer }\end{array}$ & 6 & Yes & No & Yes & No & No & No & No & Yes & Yes & Yes \\
\hline Renal & 11 & Yes & Yes & No & No & Yes & Yes & No & Yes & No & No \\
\hline Sarcoma & 11 & Yes & No & No & No & No & No & No & No & No & No \\
\hline Skin cancer & 1 & Yes & Yes & No & No & No & No & No & No & No & No \\
\hline $\begin{array}{c}\text { Testicular } \\
\text { cancer }\end{array}$ & 1 & Yes & Yes & Yes & Yes & No & Yes & No & No & Yes & No \\
\hline
\end{tabular}

Expression data were obtained from the Oncomine Cancer Microarray database. Genes which were overexpressed in cancer cell samples in comparison with their normal counterpart are indicated in this table.

presence of the two forms of extracellular sulfatases in human cancer have not been described and could be of interest.

\section{Conclusions}

The secretion of SULF1 and SULF2 raises the possibility for cancer cells to remodel the extra-cellular matrix in their environment, thereby affecting their development and/or the neighbouring host cells. A strong parallelism can be proposed with heparanase, an enzyme able to cleave HS chains, generating bioactive fragments and leading to protumorigenic effects in various models of cancer and metastatic processes [54]. However, if heparanase is clearly associated to protumorigenic effects, contradictory observations have been made concerning SULF1 and SULF2 contribution in human neoplasia, as we have discussed in this article. These differences could be explained by the various components of tumour microenvironment that can be targeted by SULF1 and SULF2. In addition, most of studies have explored the expression of these sulfatases by cancer cells but, as secreted enzymes, their production by other cell types in cancer stroma could have major effects on signaling mediated by HSPGs. Besides, the possibility of splicing variants could partially explain the different consequences of the surexpression of these proteins in neoplasia. Finally, targeting SULF1 and/or SULF2 could be interesting strategies to develop novel cancer therapies.

\section{List of abbreviations used}

Akt: $v$-akt murine thymoma viral oncogene homolog 1; b2M: beta 2 microglobulin; FGF: fibroblast growth factor; GF: growth factor; GPI: growth proliferation index; HB-EGF: heparin-binding epidermal growth factor-like growth factor; HCC: hepatocellular carcinoma; HDAC: histone deacetylase; HGF: hepatocyte growth factor; HMCL: human myeloma cell line; HRS: high risk score; HS: heparan sulphate; HSPG: heparan sulfate protéoglycane; HUVEC: human umbilical vein endothelial cells; MAPK: mitogen-activated protein kinase; MM: multiple myeloma; MS: MMSET group; MYC: v-myc myelocytomatosis viral oncogene homolog; OS: overall survival; SCCHN: head and neck squamous cell carcinoma; SCID: severe combined immunodéficiente; sh-RNA: short-hairpin RNA; SULF1: sulfatase 1; SULF2: sulfatase 2; VEGF: vascular endothelial growth factor; Wnt: wingless-type MMTV integration site family.

\section{Acknowledgements}

This work was supported by grants from the Ligue Nationale Contre le Cancer (équipe labellisée 2009), Paris, France, from INCA (nRPT09001FFA) and from MSCNET European strep ( ${ }^{\circ}$ E06005FF), the Hopp-Foundation. No financial interest/relationships with financial interest relating to the topic of this article have been declared. 


\section{Author details}

${ }^{1}$ INSERM U847, Institut de Recherche en Biothérapie, CHRU de Montpellier, France. ${ }^{2}$ Laboratoire Central d'Hématologie, CHRU de Montpellier, France. ${ }^{3}$ UFR de Médecine, Université de Montpellier, France. ${ }^{4}$ Medizinische Klinik und Poliklinik V, Heidelberg, Germany. ${ }^{5}$ Nationales Centrum für Tumorerkrankungen, INF350, Heidelberg, Germany.

\section{Authors' contributions}

CB designed the study, supported data analysis and wrote the paper JM was involved in the study design and supported data analysis. JFS and DH participated in the design of the study.

BK is the senior investigator who designed research and wrote the paper. All authors read and approved the final manuscript.

\section{Competing interests}

The authors declare that they have no competing interests.

Received: 29 October 2010 Accepted: 21 May 2011

Published: 21 May 2011

\section{References}

1. Sasisekharan R, Venkataraman G: Heparin and heparan sulfate: biosynthesis, structure and function. Curr Opin Chem Biol 2000, 4:626-631.

2. Morimoto-Tomita M, Uchimura K, Werb Z, Hemmerich S, Rosen SD: Cloning and characterization of two extracellular heparin-degrading endosulfatases in mice and humans. J Biol Chem 2002, 277:49175-49185.

3. Holst CR, Bou-Reslan H, Gore BB, Wong K, Grant D, Chalasani S, Carano RA, Frantz GD, Tessier-Lavigne M, Bolon B, et al: Secreted sulfatases Sulf1 and Sulf2 have overlapping yet essential roles in mouse neonatal survival. PLoS One 2007, 2:e575.

4. Kalus I, Salmen B, Viebahn C, von Figura K, Schmitz D, D'Hooge R, Dierks T: Differential involvement of the extracellular 6-O-endosulfatases Sulf1 and Sulf2 in brain development and neuronal and behavioural plasticity. J Cell Mol Med 2009, 13:4505-4521.

5. Rhodes DR, Yu J, Shanker K, Deshpande N, Varambally R, Ghosh D, Barrette T, Pandey A, Chinnaiyan AM: ONCOMINE: a cancer microarray database and integrated data-mining platform. Neoplasia 2004, 6:1-6.

6. Assou S, Le Carrour T, Tondeur S, Strom S, Gabelle A, Marty S, Nadal L, Pantesco V, Reme T, Hugnot JP, et al: A meta-analysis of human embryonic stem cells transcriptome integrated into a web-based expression atlas. Stem Cells 2007, 25:961-973.

7. Elfilali A, Lair S, Verbeke C, La Rosa P, Radvanyi F, Barillot E: ITTACA: a new database for integrated tumor transcriptome array and clinical data analysis. Nucleic Acids Res 2006, 34:D613-616.

8. Lai J, Chien J, Staub J, Avula R, Greene EL, Matthews TA, Smith DI, Kaufmann SH, Roberts LR, Shridhar V: Loss of HSulf-1 up-regulates heparin-binding growth factor signaling in cancer. J Biol Chem 2003, 278:23107-23117.

9. Staub J, Chien J, Pan Y, Qian X, Narita K, Aletti G, Scheerer M, Roberts LR, Molina J, Shridhar V: Epigenetic silencing of HSulf-1 in ovarian cancer: implications in chemoresistance. Oncogene 2007, 26:4969-4978.

10. Liu P, Khurana A, Rattan R, He X, Kalloger S, Dowdy S, Gilks B, Shridhar V: Regulation of HSulf-1 expression by variant hepatic nuclear factor 1 in ovarian cancer. Cancer Res 2009, 69:4843-4850.

11. Lai JP, Thompson JR, Sandhu DS, Roberts LR: Heparin-degrading sulfatases in hepatocellular carcinoma: roles in pathogenesis and therapy targets. Future Oncol 2008, 4:803-814.

12. Chen Z, Fan JQ, Li J, Li QS, Yan Z, Jia XK, Liu WD, Wei LJ, Zhang FZ, Gao H, et al: Promoter hypermethylation correlates with the Hsulf-1 silencing in human breast and gastric cancer. Int J Cancer 2009, 124:739-744.

13. Zhao H, Ramos CF, Brooks JD, Peehl DM: Distinctive gene expression of prostatic stromal cells cultured from diseased versus normal tissues. J Cell Physiol 2007, 210:111-121.

14. Lai JP, Chien J, Strome SE, Staub J, Montoya DP, Greene EL, Smith DI, Roberts LR, Shridhar V: HSulf-1 modulates HGF-mediated tumor cell invasion and signaling in head and neck squamous carcinoma. Oncogene 2004, 23:1439-1447.

15. Ai X, Kitazawa T, Do AT, Kusche-Gullberg M, Labosky PA, Emerson CP Jr: SULF1 and SULF2 regulate heparan sulfate-mediated GDNF signaling for esophageal innervation. Development 2007, 134:3327-3338.
16. Langsdorf A, Do AT, Kusche-Gullberg M, Emerson CP Jr, Ai X: Sulfs are regulators of growth factor signaling for satellite cell differentiation and muscle regeneration. Dev Biol 2007, 311:464-477.

17. Lamanna WC, Frese MA, Balleininger M, Dierks T: Sulf loss influences N-, 2O-, and 6-O-sulfation of multiple heparan sulfate proteoglycans and modulates fibroblast growth factor signaling. J Biol Chem 2008, 283:27724-27735.

18. Lai JP, Chien JR, Moser DR, Staub JK, Aderca I, Montoya DP, Matthews TA, Nagorney DM, Cunningham JM, Smith DI, et al: hSulf1 Sulfatase promotes apoptosis of hepatocellular cancer cells by decreasing heparin-binding growth factor signaling. Gastroenterology 2004, 126:231-248.

19. Narita K, Chien J, Mullany SA, Staub J, Qian X, Lingle WL, Shridhar V: Loss of HSulf-1 expression enhances autocrine signaling mediated by amphiregulin in breast cancer. J Biol Chem 2007, 282:14413-14420.

20. Dai Y, Yang Y, MacLeod V, Yue X, Rapraeger AC, Shriver Z, Venkataraman G, Sasisekharan R, Sanderson RD: HSulf-1 and HSulf-2 are potent inhibitors of myeloma tumor growth in vivo. J Biol Chem 2005, 280:40066-40073.

21. Narita K, Staub J, Chien J, Meyer K, Bauer M, Friedl A, Ramakrishnan S, Shridhar V: HSulf-1 inhibits angiogenesis and tumorigenesis in vivo. Cancer Res 2006, 66:6025-6032.

22. Lai JP, Yu C, Moser CD, Aderca I, Han T, Garvey TD, Murphy LM, GarrityPark MM, Shridhar V, Adjei AA, Roberts LR: SULF1 inhibits tumor growth and potentiates the effects of histone deacetylase inhibitors in hepatocellular carcinoma. Gastroenterology 2006, 130:2130-2144.

23. Lai JP, Sandhu DS, Moser CD, Cazanave SC, Oseini AM, Shire AM, Shridhar V, Sanderson SO, Roberts LR: Additive effect of apicidin and doxorubicin in sulfatase 1 expressing hepatocellular carcinoma in vitro and in vivo. J Hepatol 2009, 50:1112-1121.

24. Lemjabbar-Alaoui $H$, van Zante $A$, Singer MS, Xue $Q$, Wang $Y Q$, Tsay D, He B, Jablons DM, Rosen SD: Sulf-2, a heparan sulfate endosulfatase, promotes human lung carcinogenesis. Oncogene 29:635-646.

25. Sethi JK, Vidal-Puig A: Wnt signalling and the control of cellular metabolism. Biochem J 427:1-17.

26. Morimoto-Tomita M, Uchimura K, Bistrup A, Lum DH, Egeblad M, Boudreau N, Werb Z, Rosen SD: Sulf-2, a proangiogenic heparan sulfate endosulfatase, is upregulated in breast cancer. Neoplasia 2005 , 7:1001-1010.

27. Bret C, Hose D, Reme T, Sprynski AC, Mahtouk K, Schved JF, Quittet P, Rossi JF, Goldschmidt H, Klein B: Expression of genes encoding for proteins involved in heparan sulphate and chondroitin sulphate chain synthesis and modification in normal and malignant plasma cells. $\mathrm{Br} J$ Haematol 2009, 145:350-368.

28. Zhan F, Huang Y, Colla S, Stewart JP, Hanamura I, Gupta S, Epstein J, Yaccoby S, Sawyer J, Burington B, et al: The molecular classification of multiple myeloma. Blood 2006, 108:2020-2028.

29. Sprynski AC, Hose D, Caillot L, Reme T, Shaughnessy JD Jr, Barlogie B, Seckinger A, Moreaux J, Hundemer M, Jourdan M, et al: The role of IGF-1 as a major growth factor for myeloma cell lines and the prognostic relevance of the expression of its receptor. Blood 2009, 113:4614-4626

30. Shaughnessy JD Jr, Zhan F, Burington BE, Huang Y, Colla S, Hanamura I, Stewart JP, Kordsmeier B, Randolph C, Williams DR, et al: A validated gene expression model of high-risk multiple myeloma is defined by deregulated expression of genes mapping to chromosome 110.1182/ blood-2006-07-038430. Blood 2007, 109:2276-2284.

31. Hose D, Reme T, Hielscher T, Moreaux J, Messner T, Seckinger A, Benner A, Shaughnessy JD Jr, Barlogie B, Zhou Y, et al: Proliferation is a central independent prognostic factor and target for personalized and riskadapted treatment in multiple myeloma. Haematologica 2011, 96:87-95.

32. Decaux O, Lode L, Magrangeas F, Charbonnel C, Gouraud W, Jezequel P, Attal M, Harousseau JL, Moreau P, Bataille R, et al: Prediction of survival in multiple myeloma based on gene expression profiles reveals cell cycle and chromosomal instability signatures in high-risk patients and hyperdiploid signatures in low-risk patients: a study of the Intergroupe Francophone du Myelome. J Clin Oncol 2008, 26:4798-4805.

33. Moreaux J, Hose D, Reme T, Jourdan E, Hundemer M, Legouffe E, Moine $P$, Bourin P, Moos M, Corre J, et al: CD200 is a new prognostic factor in multiple myeloma. Blood 2006, 108:4194-4197.

34. Zhan F, Huang Y, Colla S, Stewart JP, Hanamura I, Gupta S, Epstein J, Yaccoby S, Sawyer J, Burington B, et al: The molecular classification of multiple myeloma 10.1182/blood-2005-11-013458. Blood 2006, 108:2020-2028. 
35. Lai JP, Sandhu DS, Yu C, Han T, Moser CD, Jackson KK, Guerrero RB, Aderca I, Isomoto H, Garrity-Park MM, et al: Sulfatase 2 up-regulates glypican 3, promotes fibroblast growth factor signaling, and decreases survival in hepatocellular carcinoma. Hepatology 2008, 47:1211-1222.

36. Nawroth $R$, van Zante A, Cervantes S, McManus M, Hebrok M, Rosen SD: Extracellular sulfatases, elements of the Wnt signaling pathway, positively regulate growth and tumorigenicity of human pancreatic cancer cells. PLoS One 2007, 2:e392.

37. Johansson FK, Goransson H, Westermark B: Expression analysis of genes involved in brain tumor progression driven by retroviral insertional mutagenesis in mice. Oncogene 2005, 24:3896-3905.

38. Moussay E, Palissot V, Vallar L, Poirel HA, Wenner T, El-Khoury V, Aouali N, van Moer K, Leners B, Bernardin F, et al: Determination of genes and microRNAs involved in the resistance to fludarabine in vivo in chronic lymphocytic leukemia. Mol Cancer 9:115.

39. Rosen SD, Lemjabbar-Alaoui H: Sulf-2: an extracellular modulator of cell signaling and a cancer target candidate. Expert Opin Ther Targets 2010, 14:935-949.

40. Riker Al, Enkemann SA, Fodstad O, Liu S, Ren S, Morris C, Xi Y, Howell P, Metge B, Samant RS, et al: The gene expression profiles of primary and metastatic melanoma yields a transition point of tumor progression and metastasis. BMC Med Genomics 2008, 1:13

41. Galamb O: mRNA expression analysis and classification of colonic biopsy samples using oligonucleotide and cDNA microarray techniques. Orv Hetil 2008, 149:1373-1377.

42. Skotheim RI, Lind GE, Monni O, Nesland JM, Abeler VM, Fossa SD, Duale N, Brunborg G, Kallioniemi O, Andrews PW, Lothe RA: Differentiation of human embryonal carcinomas in vitro and in vivo reveals expression profiles relevant to normal development. Cancer Res 2005, 65:5588-5598.

43. Wurmbach E, Chen YB, Khitrov G, Zhang W, Roayaie S, Schwartz M, Fiel I, Thung S, Mazzaferro V, Bruix J, et al: Genome-wide molecular profiles of HCV-induced dysplasia and hepatocellular carcinoma. Hepatology 2007, 45:938-947.

44. Durig J, Bug S, Klein-Hitpass L, Boes T, Jons T, Martin-Subero Jl, Harder L, Baudis M, Duhrsen $U$, Siebert R: Combined single nucleotide polymorphism-based genomic mapping and global gene expression profiling identifies novel chromosomal imbalances, mechanisms and candidate genes important in the pathogenesis of T-cell prolymphocytic leukemia with inv(14)(q11q32). Leukemia 2007, 21:2153-2163.

45. Stegmaier K, Ross KN, Colavito SA, O'Malley S, Stockwell BR, Golub TR: Gene expression-based high-throughput screening(GE-HTS) and application to leukemia differentiation. Nat Genet 2004, 36:257-263.

46. Gumz ML, Zou H, Kreinest PA, Childs AC, Belmonte LS, LeGrand SN, Wu K, Luxon BA, Sinha M, Parker AS, et al: Secreted frizzled-related protein 1 loss contributes to tumor phenotype of clear cell renal cell carcinoma. Clin Cancer Res 2007, 13:4740-4749.

47. Bhattacharjee A, Richards WG, Staunton J, Li C, Monti S, Vasa P, Ladd C, Beheshti J, Bueno R, Gillette M, et al: Classification of human lung carcinomas by mRNA expression profiling reveals distinct adenocarcinoma subclasses. Proc Natl Acad Sci USA 2001, 98:13790-13795.

48. Huang E, Cheng SH, Dressman H, Pittman J, Tsou MH, Horng CF, Bild A, Iversen ES, Liao M, Chen CM, et al: Gene expression predictors of breast cancer outcomes. Lancet 2003, 361:1590-1596.

49. Sorlie T, Tibshirani R, Parker J, Hastie T, Marron JS, Nobel A, Deng S, Johnsen H, Pesich R, Geisler S, et al: Repeated observation of breast tumor subtypes in independent gene expression data sets. Proc Natl Acad Sci USA 2003, 100:8418-8423.

50. Abiatari I, Kleeff J, Li J, Felix K, Buchler MW, Friess H: Hsulf-1 regulates growth and invasion of pancreatic cancer cells. J Clin Pathol 2006 59:1052-1058.

51. Sahota AP, Dhoot GK: A novel SULF1 splice variant inhibits Wnt signalling but enhances angiogenesis by opposing SULF1 activity. Exp Cell Res 2009, 315:2752-2764.

52. Adamsen BL, Kravik KL, Clausen OP, De Angelis PM: Apoptosis, cell cycle progression and gene expression in TP53-depleted HCT116 colon cancer cells in response to short-term 5-fluorouracil treatment. Int J Oncol 2007, 31:1491-1500.

53. Chau BN, Diaz RL, Saunders MA, Cheng C, Chang AN, Warrener $P$, Bradshaw J, Linsley PS, Cleary MA: Identification of SULF2 as a novel transcriptional target of p53 by use of integrated genomic analyses. Cancer Res 2009, 69:1368-1374.
54. Vlodavsky I, Elkin M, Abboud-Jarrous G, Levi-Adam F, Fuks L, Shafat I, Ilan N: Heparanase: one molecule with multiple functions in cancer progression. Connect Tissue Res 2008, 49:207-210.

55. Onken MD, Worley LA, Ehlers JP, Harbour JW: Gene expression profiling in uveal melanoma reveals two molecular classes and predicts metastatic death. Cancer Res 2004, 64:7205-7209.

doi:10.1186/1479-5876-9-72

Cite this article as: Bret et al:: SULFs in human neoplasia: implication as progression and prognosis factors. Journal of Translational Medicine 2011 9:72.

\section{Submit your next manuscript to BioMed Central and take full advantage of:}

- Convenient online submission

- Thorough peer review

- No space constraints or color figure charges

- Immediate publication on acceptance

- Inclusion in PubMed, CAS, Scopus and Google Scholar

- Research which is freely available for redistribution

Submit your manuscript at www.biomedcentral.com/submit
C) Biomed Central 